\title{
Embarazo y Síndrome de Evans: a propósito de un caso
}

\author{
Santiago Artucio ${ }^{1}$, Rosina Silva Ledesmaํㅡ, Sebastián Ben², Verónica Fiol ${ }^{3}$, Francisco Cóppola ${ }^{4 a, b}$
}

\section{RESUMEN}

El síndrome de Evans es la coexistencia de trombocitopenia autoinmune con anemia hemolítica autoinmune. Raramente se presenta durante el curso de un embarazo. Esto dificulta las opciones terapéuticas, dado que algunos de los agentes utilizados para el tratamiento son teratogénicos. Los efectos del síndrome de Evans en el feto y recién nacido se desconocen dado el escaso número de casos publicados.

Presentamos el caso de una paciente con diagnóstico preconcepcional de síndrome de Evans, que presenta una crisis hemolítica en el curso de una gestación, y diagnóstico de restricción del crecimiento intrauterino (RCIU), en tratamiento en la Clínica Ginecotocológica "A" del Centro Hospitalario Pereira Rossell (CHPR), de Montevideo, Uruguay. Se analizan opciones terapéuticas y evolución, así como publicaciones previas. (Horiz Med 2015; 15(4): 67-73)

Palabras clave: Síndrome de Evans - embarazo - crisis hemolítica - restricción de crecimiento intrauterino (Fuente: DeCS BIREME).

Pregnancy and Evans'syndrome: case report

\section{ABSTRACT}

Evans' syndrome is the coexistence of autoimmune thrombocytopenia with autoimmune hemolytic anemia. It is rarely found during the course of a pregnancy. This makes treatment options more difficult, since some therapeutic drugs are teratogenic. The effects of Evans' syndrome in the fetus and newborn are unknown given the low number of reported cases.

We report the case of a patient with preconceptional diagnosis of Evans' syndrome, who develops a hemolytic crisis during the course of a pregnancy, and diagnosis of intrauterine growth restriction (IUGR), treated at Clínica Ginecotocológica "A" at the Pereira Rossell Hospital Center, in Montevideo, Uruguay. Treatment options and evolution are analyzed, as well as previous reports. (Horiz Med 2015; 15(4): 67-73)

Key words: Evans' syndrome - pregnancy - hemolytic crisis - intrauterine growth restriction. (Source: MeSH NLM).

\footnotetext{
Doctor en Medicina, Residente de Ginecotocología

Doctor en Medicina, Ginecotocólogo, Asistente de Clínica Ginecotocológica

Doctor en Medicina, Ginecotocóloga, Profesora Adjunta de Clínica Ginecotocológica

Doctor en Medicina, Ginecotocólogo, Profesor Agregado de Clínica Ginecotocológica

Clínica Ginecotocológica “A” Prof. Dr. Briozzo, Facultad de Medicina, Universidad de la República.

Centro Hospitalario Pereira Rossell, Montevideo, Uruguay.
} 


\section{INTRODUCCIÓN}

El síndrome de Evans, descrito en 1951, es la coexistencia de trombocitopenia autoinmune con anemia hemolítica autoinmune $(1,2)$, con prueba de Coombs directa positiva (3).

Puede presentarse con o sin hemorragias cutáneomucosas, a las cuales suele asociarse hipocoloración de piel y mucosas, astenia, adinamia, disnea, mareos y palpitaciones derivados de anemia aguda, así como también ictericia debido a la hemólisis con acumulación de pigmentos biliares.

Algunos casos se presentan con neutropenia, e incluso pancitopenia $(2,4)$.

Es una enfermedad hematológica de base etiopatogénica autoinmune, que cursa en empujes y remisiones (2-5).

Se presenta más frecuentemente en la edad pediátrica. Es una patología de ocurrencia rara, con frecuencia desconocida, que se estima afecta al 1,8 - $10 \%$ de los pacientes con Púrpura Trombocitopénico Idiopático (PTI) (6).

Usualmente se detectan autoanticuerpos que actúan a nivel de las células de la sangre. Más frecuentemente, la trombocitopenia autoinmune es causada por anticuerpos contra glucoproteínas de superficie plaquetaria, como llb/llla o lb/IX.

Por otro lado, la anemia hemolítica en $60-80 \%$ de los casos es causada por anticuerpos calientes, activos en temperaturas de $35-40^{\circ}$. En general, la hemólisis es extravascular y ocurre en el bazo (4).

El síndrome de Evans raramente se presenta durante el curso de un embarazo. Esto dificulta las opciones terapéuticas, dado que algunos de los agentes más frecuentemente utilizados para el tratamiento del síndrome de Evans son teratogénicos $(5,7)$.

De ser el debut, su aparición durante el embarazo nos obliga a descartar el Síndrome de HELLP caracterizado también por anemia hemolítica, plaquetopenia y elevación de enzimas hepáticas que se presenta, en general, en el contexto de un Síndrome de Preeclampsia-Eclampsia (5).
Los efectos del síndrome de Evans en el feto y recién nacido se desconocen dado el escaso número de casos publicados.

\section{REPORTE DE CASO}

Paciente de 20 años, de raza blanca, primigesta, con antecedente de síndrome de Evans diagnosticado a los 12 años de edad en el contexto de crisis hemolítica caracterizada por síndrome funcional anémico, ictericia y esplenomegalia, asociada a anemia con hemoglobina de $1,1 \mathrm{~g} / \mathrm{dL}$ que agrega plaquetopenia de $70.000 / \mathrm{mm} 3$.

Presenta múltiples empujes de su enfermedad desde el diagnóstico, con última crisis un mes previo al diagnóstico de embarazo, en tratamiento con prednisona, ciclosporina $\mathrm{A}$ y micofenolato de mofetil en control con Servicio de Hematooncología del Centro Hospitalario Pereira Rossell (CHPR), Montevideo, Uruguay.

Es captada cursando embarazo de 12 semanas de edad gestacional (EG). Al ingreso se suspende tratamiento con micofenolato de mofetil y se mantiene tratamiento con prednisona y ciclosporina A.

De la paraclínica a las 12 semanas se destaca hemoglobina de $12,1 \mathrm{~g} / \mathrm{dL}$ y recuento plaquetario de $127.000 / \mathrm{mm} 3$. Embarazo bien tolerado con rutinas obstétricas, ecografía estructural y ecocardiograma normales.

Cursando 31 semanas ingresa por crisis hemolítica con anemia severa, con palidez cutáneo-mucosa intensa, astenia, adinamia, con ictericia universal.

La paraclínica muestra hemoglobina $4,7 \mathrm{~g} / \mathrm{dL}$, plaquetas $110000 / \mathrm{mm} 3$, bilirrubina total de $4,0 \mathrm{mg} / \mathrm{dL}$ a predominio de indirecta, y lactato deshidrogenasa de 590. Se instaura tratamiento con metilprednisolona ( $1 \mathrm{mg} / \mathrm{kg} / \mathrm{día}$ ), continuando con corticoterapia a altas dosis; transfusión de 1 volumen de glóbulos rojos y gammaglobulina hiperinmune intravenosa $25 \mathrm{~g}$ postransfusión.

Cursando 32 semanas de edad gestacional, se realiza diagnóstico clínico de restricción del crecimiento intrauterino (RCIU) con altura uterina discordante en menos. La ecografía obstétrica confirma RCIU 
con oligoamnios y peso fetal estimado de $1330 \mathrm{~g}$. La ecografía obstétrica confirma feto en presentación podálica.

El estudio Doppler fetoplacentario informa resistencias fetoplacentarias y maternas normales, estado hemodinámico fetal bueno, con velocidad máxima de la arteria cerebral media compatible con hemoglobina fetal calculada de $13,75 \mathrm{~g} / \mathrm{dL}$ (no anemia fetal).

Se descartan otras causas frecuentes de restricción del crecimiento, como ser estados hipertensivos inducidos por el embarazo, otras patologías maternas sistémicas, consumo de tóxicos, desnutrición materna e infecciosas.

Se decide interrupción de la gravidez por el riesgo de mortalidad materna por anemia severa que no responde de manera adecuada al tratamiento médico propuesto, y el riesgo aumentado de muerte fetal intrauterina por RCIU secundario a la misma.

Se decide realizar operación cesárea de urgencia por riesgo materno y fetal, así como situación obstétrica con feto en podálica. Se realiza el procedimiento bajo anestesia general.

Se obtiene un recién nacido vivo, de sexo femenino, con peso al nacer de $1460 \mathrm{~g}$, con score de Apgar de
2 - 4 - 7. Se realizó en el intraoperatorio transfusión de 2 volúmenes de glóbulos rojos.

Con buena evolución postoperatoria, se otorga el alta a hematooncología al tercer día de postoperatorio.

De la paraclínica al alta se destaca hemoglobina de $9,6 \mathrm{~g} / \mathrm{dL}$, recuento plaquetario de $81.000 / \mathrm{mm} 3$.

El recién nacido presenta ictericia. El test de Coombs directo fue negativo. Se realiza fototerapia intensiva y requiere exanguinotransfusión a las 16 horas de vida con bilirrubina total de $5,1 \mathrm{mg} / \mathrm{dL}$ (directa 0,91 - indirecta 4,16).

Se administra inmunoglobulina intravenosa 1 dosis previa a exanguinotransfusión y otra luego de la misma.

Requiere fototerapia por 3 días. Se evidencia descenso de hematocrito de $56 \%$ a $28 \%$ al $7 \mathrm{mo}$ día de vida, por lo que se realiza transfusión de glóbulos rojos (hemoglobina desciende de 18,6 g/ $\mathrm{dL}$ a $9,9 \mathrm{~g} / \mathrm{dL}$ ).

Presenta plaquetopenia que requiere administración de 2 concentrados plaquetarios.

Tabla 1. Evolución de paraclínica materna durante ingreso hospitalario y tratamiento mediante transfusiones. [Unidades: Hemoglobina (g/ $\mathrm{dL}$ ), Hematocrito (\%), Leucocitos (x103/mm3), Plaquetas (x103/mm3), Bilirrubina Total (mg/dL), Bilirrubina Directa (mg/dL), TGO (UI/L), $\operatorname{TGP}(\mathrm{UI} / \mathrm{L})$, LDH (UI/L)]

\begin{tabular}{|c|c|c|c|c|c|c|c|c|c|}
\hline Fecha & Dia 1 & Dia 2 & Dia 3 & Dia 4 & Dia 5 & Dia 6 & Dia 10 & Dia 11 & Dia 11 (post plaq) \\
\hline Hemoglobina & 4.7 & 5.7 & 6.2 & 6.5 & 5.8 & 6.3 & & 7.9 & \\
\hline Hematocrito & 14.2 & 17.3 & 18.7 & 19.5 & 17 & 18 & & 22.7 & \\
\hline Leucocitos & 8.1 & 8.4 & 6.1 & 5.8 & 5.2 & 6.8 & & 15.9 & \\
\hline Plaquetas & 110 & 110 & 74 & 95 & 76 & 83 & & 70 & 100 \\
\hline Bilirrubina T & 4 & 3.7 & & & & 5.6 & & & \\
\hline Bilirrubina D & 1.33 & 1.06 & & & & 1.07 & & & \\
\hline TGO & 26 & 25 & & & & 18 & & & \\
\hline TGP & 24 & 31 & & & & 530 & & & \\
\hline LDH & 890 & 624 & & & & & & & \\
\hline \multirow[t]{2}{*}{ Transfusiones } & & $1 \mathrm{Vol}$ & & & & & $2 \mathrm{Vol}$ & $1 \mathrm{Vol}$ & $2 \mathrm{Vol}$ \\
\hline & & GR & & & & & GR & Plaq & GR \\
\hline
\end{tabular}




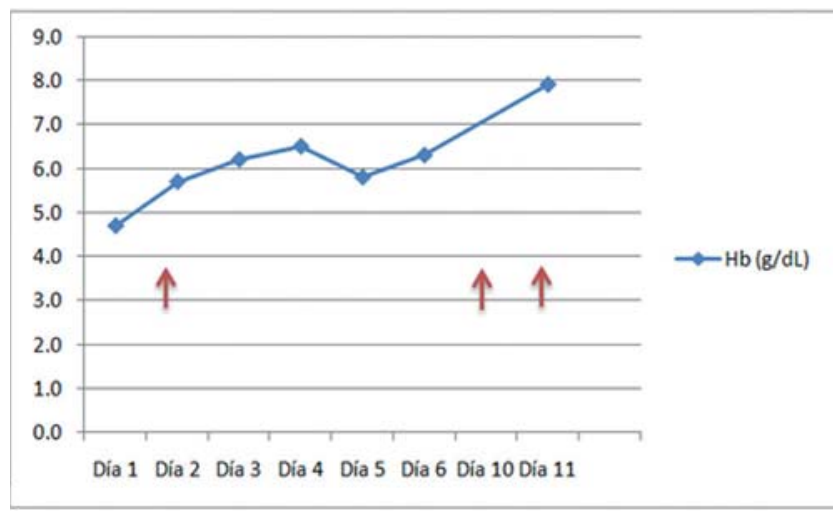

Figura 1. Concentración de Hemoglobina materna en internación. En rojo, momentos en que recibe transfusión de GR (ver cantidades en Tabla 1).

Tabla 2. Evolución paraclínica del neonato durante internación. [Unidades: Bilirrubina total (mg/dL), Hematocrito (\%), Hemoglobina (g/dL), Plaquetas $\left.\left(\times 10^{3} / \mathrm{mm}^{3}\right)\right]$

\begin{tabular}{|l|l|l|l|l|l|}
\hline Horas de vida & $\mathbf{4}$ & $\mathbf{1 6}$ & $\mathbf{7 2}$ & $\mathbf{9 6}$ & $\mathbf{1 6 8}$ \\
\hline $\begin{array}{l}\text { Bilirrubina } \\
\text { total }\end{array}$ & 9,6 & 5,1 & 3,8 & 6,4 & 3,5 \\
\hline Hematocrito & 54,6 & 40,8 & 44,1 & 41,5 & 28,6 \\
\hline Hemoglobina & 18,6 & 13,9 & 14,4 & 13,5 & 9,9 \\
\hline Plaquetas & 238 & 116 & 102 & 83 & 141 \\
\hline & & & & & \\
\hline
\end{tabular}

\section{DISCUSIÓN}

El síndrome de Evans es una patología hematológica de base autoinmune extremadamente rara, en la que coexisten trombocitopenia y anemia hemolítica autoinmunes (1-5).

Dado que esta enfermedad de por sí no genera alteraciones de la fertilidad, aquellas pacientes que lo presentan y se encuentran en edad reproductiva pueden cursar un embarazo.

Dado que la medicación utilizada para el manejo de base de esta patología puede presentar efectos teratogénicos $(5,7)$, su manejo debe ser llevado a cabo por especialistas.

Por otro lado, su diagnóstico se realiza en la mayoría de los casos en la edad pediátrica (6), por lo que se debe ofrecer opciones anticonceptivas a las pacientes al llegar a la edad reproductiva para planificar con antelación un embarazo.

Realizamos una búsqueda bibliográfica en las bases de datos de Medline (PubMed), en SciELO y en la biblioteca de salud de Cochrane a la fecha de diciembre de 2014.

No obtuvimos resultados bajo los términos "Evans syndrome" y "pregnancy" para trabajos publicados en América del Sur.

En América Latina ha sido publicado un reporte de caso de debut de síndrome de Evans en embarazo en Costa Rica (6), por lo que nuestro caso es el segundo reportado en la región.

En el caso de nuestra paciente, se presenta con diagnóstico de síndrome de Evans previo al embarazo, desde la edad pediátrica. Por esto, no se plantean otros diagnósticos diferenciales como causa de su anemia y trombocitopenia en el embarazo.

En aquellas pacientes que no presentan diagnóstico de síndrome de Evans previo al embarazo es extremadamente difícil hacer este diagnóstico durante la gestación.

Se debe descartar la presencia de otras causas de trombocitopenia, anemia, e incluso enfermedad hepática gestacional más frecuentes.

Se debe descartar la concomitancia de un estado hipertensivo del embarazo, dado que orientará fuertemente a la presencia de un síndrome HELLP (5). En éste caso clínico la paciente cursó con cifras de presión arterial normales.

Es de suma importancia que estas pacientes sean debidamente asesoradas con respecto a la planificación del embarazo, dado que el tratamiento de mantenimiento de esta patología puede tener efectos abortivos o teratogénicos (micofenolato de mofetil, otros agentes inmunosupresores) $(5,7)$.

En nuestro caso la paciente fue captada al final 
del primer trimestre por lo que se realizaron 2 ecografías morfoestructurales y ecocardiograma fetal a las 19, 22 y 24 semanas respectivamente, sin evidencia de malformaciones fetales. No se observaron malformaciones en el recién nacido en la evaluación postnatal.

En nuestro caso clínico la paciente presentó una crisis hemolítica con anemia severa. El tratamiento de la crisis fue altas dosis de corticosteroides, transfusión de glóbulos rojos y gammaglobulina hiperinmune intravenosa.

El tratamiento con corticoides a altas dosis y transfusión de glóbulos roj os ha sido usado en casos de anemia hemolítica autoinmune en embarazo con buenos resultados previamente (8-11).

Salvo por publicación de casos aislados, no contamos con evidencia de estudios clínicos de tratamiento de anemia hemolítica autoinmune en el embarazo.

El tratamiento en el embarazo debe ser individualizado según la situación clínica. En pacientes no embarazadas, el tratamiento de primera línea es altas dosis de corticoides, mientras que de segunda línea es la esplenectomía, el uso de rituximab y el uso de gammaglobulina hiperinmune intravenosa $(11,12)$.

En nuestra paciente se utilizó la terapéutica disponible menos invasiva dada su condición gravídica.

A nivel feto-neonatal no se ha demostrado evidencia de efectos directos por el síndrome de Evans.

Se plantea la posibilidad de teratogenicidad vinculada al uso de fármacos teratogénicos para el tratamiento (micofenolato de mofetil, agentes inmunosupresores), que en nuestro caso se descartó de forma prenatal como fue analizado.

Por otro lado, debido a la anemia materna se pueden desarrollar complicaciones fetales, como en el caso de nuestra paciente, en la que se diagnosticó una restricción de crecimiento intrauterino en el embarazo (RCIU).
La causa de la RCIU fue la anemia materna, habiendo descartado otras causas como estados hipertensivos del embarazo, exposición a tóxicos, escaso incremento ponderal materno, entre otros. En otros casos reportados también se ha asociado el síndrome de Evans a anemia materna y restricción del crecimiento intrauterino (9).

La anemia materna se caracteriza por descenso de hemoglobina circulante, que provoca un estado de hipoxia tisular crónica y aumento del estrés oxidativo, lo cual compromete el crecimiento fetal.

Se ha demostrado que la anemia materna moderada - severa, con valores de hemoglobina menores de 8,0 - 9,0 g/dL pueden provocar RCIU (13). Por último, existe la posibilidad que anticuerpos circulantes maternos atraviesen la placenta y tengan repercusión fetal-neonatal.

En nuestro caso clínico, el neonato no presentó anemia al nacimiento, mientras que sí requirió exanguínotransfusión por ictericia. Al no presentar anemia, planteamos que la causa de la hiperbilirrubinemia es materna por pasaje transplacentario.

Posteriormente desarrolla anemia y trombocitopenia. Existe evidencia en la literatura de pasaje de anticuerpos maternos al feto por vía transplacentaria, que puede explicar esta complicación $(5,10)$. Al ser inmunomediada, se detecta anemia en el neonato día luego del nacimiento (10).

En relación a la valoración fetal, para descartar la presencia de anemia hemolítica autoinmune fetal por pasaje de anticuerpos maternos vía transplacentaria, se realizó la valoración de la velocidad máxima de flujo de la arteria cerebral media del feto, mediante velocimetría Doppler por ultrasonografía, para estimación de hemoglobina fetal.

Existe evidencia del uso de esta técnica diagnóstica para evaluación de aloinmunización materna para factor Rhesus (14). No hemos encontrado evidencia al respecto del uso de esta técnica para 
el diagnóstico prenatal en casos de pacientes con síndrome de Evans y embarazo.

Este estudio mostró un estado de no anemia, Io que fue acorde con los controles de hemoglobina y hematocrito del neonato al nacimiento.

Con respecto a la vía de finalización del embarazo, no hay evidencia de que la realización de una cesárea disminuya el riesgo de hemorragia intracraneana neonatal, aumentando en forma significativa el riesgo de hemorragia materna. Por éste motivo se recomienda que la vía de finalización sea determinada por la situación obstétrica $(11,15)$.

En el caso clínico aquí presentado, la situación obstétrica fue no favorable para parto vaginal dado que el feto se encontraba en presentación podálica.

Con respecto a la realización de anestesia epidural y el riesgo de hematoma epidural existe evidencia clínica limitada que sugiere que éste tipo de anestesia es un procedimiento seguro en casos de recuento plaquetario mayor a 100.000/ mm3 (15).

En éste caso se realizó anestesia general por presentar al momento de la interrupción un recuento plaquetario de $70.000 / \mathrm{mm} 3$.

Presentamos el caso de una paciente con diagnóstico de síndrome de Evans previo al embarazo, cursando un embarazo que se interrumpió a las 32 semanas por anemia materna y restricción del crecimiento fetal, con buena evolución posterior.

\section{Fuentes de financiamiento}

Este estudio ha sido financiado por los autores.

\section{Conflictos de interés}

Los autores declaran no tener conflicto de interés en la presentación de este artículo. 


\section{REFERENCIAS BIBLIOGRÁFICAS}

1. Evans RS, Takahashi K, Duane RT, Payne R, Lie CK. Primary thrombocytopenic purpura and acquired hemolytic anemia: evidence for common etiology. Arch Intern Med 1951;87:48 -65.

2. Arceci RJ, Hann IM, Smith OP, editors. Pediatric Hematology. 3rd ed. Massachusetts: Blackwell Publishing; 2006.

3. Dhingra KK, Jain D, Mandal S, Khurana N, Singh T, Gupta N. Evans syndrome: a study of six cases with review of literature. Hematology. 2008 Dec;13(6):356-60.

4. Norton A, Roberts I. Management of Evans syndrome. $\mathrm{Br} \mathrm{J}$ Haematol. 2006 Jan;132(2):125-37.

5. Lefkou E, Nelson-Piercy C, Hunt BJ. Evans' syndrome in pregnancy: a systematic literature review and two new cases. Eur J Obstet Gynecol Reprod Biol. 2010 Mar;149(1):10-7.

6. Barboza-Retana J, Bogantes-Ledezma P, Carballo-Gutiérrez W, Díaz-Camacho J. Evans Syndrome of Sudden Onset in Pregnancy. Acta méd. costarric. 2011 Oct-Dec; 53(4).

7. Anderka MT, Lin AE, Abuelo DN, Mitchell AA, Rasmussen SA. Reviewing the evidence for mycophenolate mofetil as a new teratogen: case report and review of the literature. Am J Med Genet A. 2009 Jun;149A(6):1241-8.

8. Baumann R, Rubin H. Autoimmune hemolytic anemia during pregnancy with hemolytic Disease in the newborn. Blood. 1973, Feb; 41 (2)

9. Hernández-Salazar E, Martínez-Abundis E, González-Ortiz M. Evans' syndrome, pregnancy and preeclampsia. Ginecol Obstet Mex. 2001 Feb; 69: 88-92.
10. Passi GR, Kriplani A, Pati HP, Choudhry VP. Isoimmune hemolysis in an infant due to maternal Evans' syndrome. Indian J Pediatr. 1997 Nov-Dec;64(6):893-5

11. Phupong V, Sareepapong W, Witoonpanich P. Evans syndrome and pregnancy: a case report. BJOG. 2004 Mar;111(3):274-6

12. Lechner $\mathrm{K}$, Jäger U. How I treat autoimmune hemolytic anemias in adults. Blood. 2010, Sept 16; 116 (11)

13. Kozuki N, Lee AC, Katz J; Child Health Epidemiology Reference Group. Moderate

to severe, but not mild, maternal anemia is associated with increased risk of small-for-gestational-age outcomes. J Nutr. 2012 Feb;142(2):358-62.

14. Mari $\mathrm{G}$ et al. Noninvasive diagnosis by Doppler ultrasonography of fetal anemia due to maternal red-cell alloimmunization. $\mathrm{N}$ Engl J Med. 2000 Jan 6;342(1):9-14.

15. "ACOG practice bulletin: thrombocytopenia in pregnancy. Number 6, September 1999. Clinical management guidelines for obstetrician-gynecologists. American College of Obstetricians and Gynecologists," International Journal of Gynecology and Obstetrics, vol. 67, no. 2, pp. 117-128, 1999.

\section{Correspondencia:}

\author{
Santiago Artucio \\ Dirección: 26 de Marzo 3486, Montevideo - Uruguay \\ Teléfono: 59899582830 \\ Correo electrónico: sartucio@gmail.com
}

